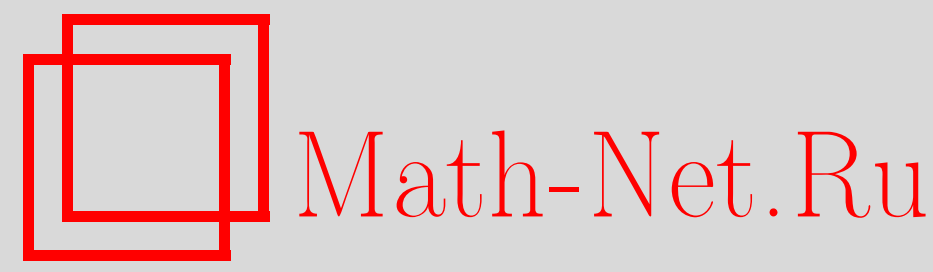

Нгуен Мань Хунг, Асимптотика решений первой краевой задачи для сильно гиперболических систем вблизи конической точки границы области, Матем. сб., 1999, том 190, номер 7, 103-126

DOI: https://doi.org/10.4213/sm415

Использование Общероссийского математического портала Math-Net.Ru подразумевает, что вы прочитали и согласны с пользовательским соглашением

http://www . mathnet.ru/rus/agreement

Параметры загрузки:

IP : 3.95 .254 .165

26 апреля 2023 г., 14:05:16 


\title{
Асимптотика решений первой краевой задачи для сильно гиперболических систем вблизи конической точки границы области
}

\begin{abstract}
В работе устанавливается теорема существования и единственности обобщенного решения первой начально-краевой задачи для сильно гиперболических систем в ограниченных областях. Рассматривается вопрос об оценках в пространствах Соболева производных по временной переменной обобшенного решения. Доказьвается, что гладкость обобщенных решений по временной переменной не зависит от структуры границы области, а зависит от коэффициентов и правой части. Получены также теоремы о гладкости обобщенного решения и его асимптотике вблизи конической точки границы области.

Библиографиия: 14 названий.
\end{abstract}

$\mathrm{K}$ настоящему моменту теория эллиптических краевых задач находится в завершенном состоянии. Это относится к задачам как в гладких областях, так и в областях, граница которых имеет особенности. В случае цилиндрической области с гладкой границей принципиальные результаты о существовании и единственности гладкого решения начально-краевой задачи для гиперболического уравнения установлены в работах Х. О. Крейсс [1], Р. Сакамото [2]. Значительно менњше результатов имеется в случае, когда область не является гладкой. Отметим работы [3]-[5]. В работах [3], [5] изучалась задача с наклонной производной для волнового уравнения в области типа двугранного угла. В работе [4] Б. А. Пламеневский изучал задачу Дирихле для волнового уравнения в соболевских пространствах с весовой нормой.

Настоящая работа посвящена специальному классу гиперболических систем "сильно гиперболическим системам". Это понятие введено О.А. Ладыженской. В работе [6] О.А. Ладыженская доказала существование и единственность обобщенного решения первой краевой задачи для таких систем без каких-либо ограничений на границу области. В монографии [7] рассмотрены основные краевые задачи для гиперболического уравнения второго порядка.

Здесь будут рассматриваться сильно гиперболические системы, имеющие порядок $2 m$ по пространственным переменным в области $\Omega \times[0, T]$, где $\Omega \in \mathbb{R}^{n}$ - ограниченная область, $T<\infty$. Предполагается, что граница $\partial \Omega$ области $\Omega$ - гладкая поверхность всюду, кроме конечного числа точек, в окрестности каждой из которых она имеет коническую структуру. Такие задачи изучались в работах [8], [9] для уравнений второго порядка, где был найден главный член асимптотического представления решения. Методы работ [7]-[9] здесь будут сушественно использованы. 
Введем необходимые обозначения: $\Omega$ - область в $\mathbb{R}^{n}, \Omega_{a, b}=\Omega \times[a, b], \Gamma_{a, b}=$ $\partial \Omega \times[a, b], \Omega_{0, b}=\Omega_{b}, \Gamma_{0, b}=\Gamma_{b}$.

Будут использованы следующие функциональные пространства.

$H^{l}(\Omega)$ - пространство комплекснозначных вектор-функций $u(x)=\left(u_{1}(x)\right.$, $\left.\ldots, u_{s}(x)\right)$, имеющих в $\Omega$ обобщенные производные до порядка $l$ включительно, такие, что

$$
\|u\|_{H^{l}(\Omega)}^{2}=\sum_{|\alpha| \leqslant l} \int_{\Omega} \sum_{i=1}^{s}\left|D^{\alpha} u_{i}\right|^{2} d x<\infty
$$

где $D^{\alpha}=\frac{\partial^{|\alpha|}}{\partial x_{1}^{\alpha_{1}} \cdots \partial x_{n}^{\alpha_{n}}},|\alpha|=\alpha_{1}+\cdots+\alpha_{n}, x=\left(x_{1}, \ldots, x_{n}\right), d x=d x_{1} \cdots d x_{n}$.

$\stackrel{\circ}{H}^{l}(\Omega)$ - подпространство $H^{l}(\Omega)$, плотным множеством в котором являются функции из $\stackrel{\circ}{C}^{\infty}(\Omega)$.

Всюду в дальнейшем функции - это комплекснозначные вектор-функции.

$H_{\beta}^{l}(\Omega)$ - пространство функций с нормой:

$$
\|u\|_{H_{\beta}^{l}(\Omega)}=\left[\sum_{|\alpha| \leqslant l} \int_{\Omega} \sum_{i=1}^{s} r^{2(\beta-l+|\alpha|)}\left|D^{\alpha} u_{i}\right|^{2} d x\right]^{1 / 2}
$$

где $r=\left(x_{1}^{2}+\cdots+x_{n}^{2}\right)^{1 / 2}$.

$H^{l, k}\left(\Omega_{a, b}\right)$ - пространство функций $u(x, t)$ таких, что $D^{\alpha} u_{i} \in L_{2}\left(\Omega_{a, b}\right),|\alpha| \leqslant l$, $\frac{\partial^{j} u_{i}}{\partial t^{j}} \in L_{2}\left(\Omega_{a, b}\right), 1 \leqslant j \leqslant k, 1 \leqslant i \leqslant s$, где

$$
\|u\|_{H^{l, k}\left(\Omega_{a, b}\right)}^{2}=\sum_{|\alpha| \leqslant l} \int_{\Omega_{a, b}}\left|D^{\alpha} u\right|^{2} d x d t+\sum_{1 \leqslant j \leqslant k} \int_{\Omega_{a, b}}\left|u_{t^{j}}\right|^{2} d x d t
$$

$\left|D^{\alpha} u\right|^{2}=\sum_{i=1}^{s}\left|D^{\alpha} u_{i}\right|^{2}, u_{t^{j}}=\frac{\partial^{j} u}{\partial t^{j}}$.

$\stackrel{\circ}{H}^{l, k}\left(\Omega_{a, b}\right)$ - подпространство $H^{l, k}\left(\Omega_{a, b}\right)$, плотным множеством в котором являются гладкие функции, равные нулю вблизи $\Gamma_{a, b}$.

$H^{l}\left(\Omega_{a, b}\right)$ - пространство функций, снабженное нормой

$$
\|u\|_{H^{l}\left(\Omega_{a, b}\right)}=\left[\sum_{|\alpha|+j \leqslant l} \int_{\Omega_{a, b}} r^{2(|\alpha|+j-l)}\left|D^{\alpha} u_{t^{j}}\right|^{2} d x d t\right]^{1 / 2} .
$$

$H_{\beta}^{l, k}\left(\Omega_{a, b}\right)$ - пространство функций, в котором норма определена так:

$$
\|u\|_{H_{\beta}^{l, k}\left(\Omega_{a, b}\right)}=\left[\sum_{|\alpha| \leqslant l} \int_{\Omega_{a, b}} r^{2(\beta+|\alpha|-l)}\left|D^{\alpha} u\right|^{2} d x d t+\sum_{j \leqslant k} \int_{\Omega_{a, b}}\left|u_{t^{j}}\right|^{2} d x d t\right]^{1 / 2} .
$$

Рассмотрим матричный дифференциальный оператор

$$
L(x, t ; D)=\sum_{|p|,|q|=1}^{m} D^{p} a_{p q}(x, t) D^{q}+\sum_{|p|=1}^{m} a_{p}(x, t) D^{p}+a(x, t),
$$


где $a_{p q}, a_{p}, a$ - ограниченные матрицы $s \times s$, причем $a_{p q}=(-1)^{|p|+|q|} a_{q p}^{*}$. Предполагается, что

$$
\sum_{|p|=|q|=m} a_{p q}(x, t) \xi^{p} \xi^{q} \zeta \bar{\zeta}>0, \quad(x, t) \in \bar{\Omega}_{T},
$$

при всех $\xi \in \mathbb{R}^{n} \backslash\{0\}$ и $\zeta \in \mathbb{C}^{s} \backslash\{0\}$.

Первая краевая задача для сильно гиперболических систем состоит в нахождении решения уравнения

$$
(-1)^{m-1} L(x, t ; D) u-u_{t t}=f, \quad(x, t) \in \Omega_{T},
$$

такого, что

$$
u=u_{t}=0 \text { при } t=0, \quad x \in \Omega
$$

и

$$
\left.D_{\nu}^{j} u\right|_{\Gamma_{T}}=0, \quad j=0, \ldots, m-1
$$

где $\nu$ - направление внешней нормали к $\Omega$.

В качестве решения задачи (0.2)-(0.4) понимается обобшенное решение. Обобщенным решением задачи (0.2)-(0.4) называется вектор-функция $u(x, t) \in$ $\stackrel{\circ}{H}^{m, 2}\left(\Omega_{T}\right)$ такая, что $u_{t} \in \stackrel{\circ}{H}^{m, 0}\left(\Omega_{T}\right),\left.u\right|_{t=0}=\left.u_{t}\right|_{t=0}=0$ и

$$
\begin{array}{r}
\int_{\Omega_{t_{1}, t_{2}}}(-1)^{m-1}\left[\sum_{|p|,|q|=1}^{m}(-1)^{|p|} a_{p q} D^{q} u \overline{D^{p} \eta}+\sum_{|p|=1}^{m} a_{p} D^{p} u \bar{\eta}+a u \bar{\eta}\right] d x d t \\
-\int_{\Omega_{t_{1}, t_{2}}} u_{t t} \bar{\eta} d x d t=\int_{\Omega_{t_{1}, t_{2}}} f \bar{\eta} d x d t
\end{array}
$$

каковы бы ни были $t_{1}, t_{2}\left(0 \leqslant t_{1}<t_{2} \leqslant T\right)$ и $\eta(x, t) \in \stackrel{\circ}{H^{m}}{ }^{0}\left(\Omega_{t_{1}, t_{2}}\right)$.

Обозначим через $B(u, v)$ билинейную форму:

$$
B(u, v)=\sum_{|p|,|q|=1}^{m}(-1)^{|p|} \int_{\Omega} a_{p q} D^{q} u \overline{D^{p} v} d x
$$

Если выполнено (0.1) и $a_{p q}$ при $|p|=|q|=m$ непрерывны в $\bar{\Omega}_{T}$, то имеет место хорошо известное неравенство Гординга [10]:

$$
(-1)^{m} B(u, u) \geqslant \mu_{0}\|u\|_{H^{m}(\Omega)}^{2}-\lambda_{0}\|u\|_{L_{2}(\Omega)}^{2},
$$

где $\mu_{0}=$ const $>0, \lambda_{0}=$ const.

В дальнейшем будет существенно использоваться следующая лемма.

ЛЕмма 1. Если и $(x, t)$ - обобщенное решение задачи $(0.2)-(0.4), f(x, t) \in$ $L_{2}\left(\Omega_{T}\right)$, то почти при всех $t \in[0, T]$ функиия $и(x, t)$ является обобщенньм решением из $\stackrel{\circ}{H}^{m}(\Omega)$ эллиптической системь

$$
(-1)^{m-1} L u=F(x, t),
$$

əде $F=u_{t t}+f$. 
ДокАЗАТЕльСТво. Пусть $\left\{\chi_{k}(x) \in \stackrel{\circ}{C}^{\infty}(\Omega), k=1,2, \ldots\right\}$ - всюду плотное в $\stackrel{\circ}{H}^{m}(\Omega)$ множество. Рассмотрим функцию $\theta(\tau) \in C^{\infty}\left(\mathbb{R}^{1}\right)$ такую, что $\theta(\tau)=0$ при $|\tau|>1 / 2$ и

$$
\int_{-\infty}^{+\infty} \theta(\tau) d \tau=1
$$

Положим в $(0.5)$

$$
\eta=h^{-1} \chi_{k}(x) \theta\left(\frac{\left|t-t^{\prime}\right|}{h}\right),
$$

где $0<t^{\prime}<T, h<\min \left(t^{\prime}, T-t^{\prime}\right)$. В результате получим

$$
\begin{aligned}
\int_{\Omega_{T}}(-1)^{m-1} & {\left[\sum_{|p|,|q|=1}^{m}(-1)^{|p|} a_{p q} D^{q} u \overline{D^{p} \chi_{k}}+\sum_{|p|=1}^{m} a_{p} D^{p} u \overline{\chi_{k}}+a u \overline{\chi_{k}}\right] } \\
\times & h^{-1} \theta\left(\frac{\left|t-t^{\prime}\right|}{h}\right) d x d t=\int_{\Omega_{T}}\left[u_{t t} \overline{\chi_{k}}+f \overline{\chi_{k}}\right] h^{-1} \theta\left(\frac{\left|t-t^{\prime}\right|}{h}\right) d x d t
\end{aligned}
$$

т.е. осреднение функции

$$
\begin{array}{r}
\xi(t)=(-1)^{m-1} \int_{\Omega}\left[\sum_{|p|,|q|=1}^{m}(-1)^{|p|} a_{p q} D^{q} u \overline{D^{p} \chi_{k}}+\sum_{|p|=1}^{m} a_{p} D^{p} u \overline{\chi_{k}}+a u \overline{\chi_{k}}\right] d x \\
-\int_{\Omega}\left[u_{t t} \overline{\chi_{k}}+f \overline{\chi_{k}}\right] d x, \quad \varepsilon<t<T-\varepsilon,
\end{array}
$$

равно нулю. Отсюда следует, что $\xi(t)=0$ на $(0, T) \backslash E_{k}$, где mes $E_{k}=0$, причем $E_{k}$ зависит от $k$. Пусть $E=\bigcup_{k=1}^{\infty} E_{k}$. Тогда $\xi(t)=0$ на $(0, T) \backslash E$. Из плотности $\left\{\chi_{k}(x)\right\}_{k=1}^{\infty}$ в $\stackrel{\circ}{H}{ }^{m}(\Omega)$ следует, что

$(-1)^{m-1} \int_{\Omega}\left[\sum_{|p|,|q|=1}^{m}(-1)^{|p|} a_{p q} D^{q} u \overline{D^{p} \chi}+\sum_{|p|=1}^{m} a_{p} D^{p} u \bar{\chi}+a u \bar{\chi}\right] d x=\int_{\Omega}\left[u_{t t}+f\right] \bar{\chi} d x$

для любой $\chi(x) \in \stackrel{\circ}{H}^{m}(\Omega)$ и $t \in(0, T) \backslash E$, т.е.

$$
(-1)^{m-1} L u=F(x, t)
$$

по определению обобшенного решения эллиптических систем. Лемма доказана.

\section{§1. Существование и единственность решения первой краевой задачи}

Tеорема 1.1. Пусть $a_{p q} \in C\left(\bar{\Omega}_{T}\right) n p u|p|=|q|=m,\left|\frac{\partial^{2} a_{p q}}{\partial t^{2}}\right|,\left|\frac{\partial a_{p q}}{\partial t}\right|,\left|\frac{\partial a_{p}}{\partial t}\right|$, $\left|\frac{\partial a}{\partial t}\right|$ ограничень при $1 \leqslant|p|,|q| \leqslant m$. Пусть, кроме того, $f \in L_{2}\left(\Omega_{T}\right)$, 
$f_{t} \in L_{2}\left(\Omega_{T}\right), f(x, 0)=0$. Тогда задача $(0.2)-(0.4)$ имеет единственное обобщенное решение и

$$
\|u\|_{H^{m, 2}\left(\Omega_{T}\right)}^{2} \leqslant C\left[\|f\|_{L_{2}\left(\Omega_{T}\right)}^{2}+\left\|f_{t}\right\|_{L_{2}\left(\Omega_{T}\right)}^{2}\right], \quad C=\text { const } .
$$

Теорема 1.1 будет доказана методом Галеркина. О.А. Ладыженская [7] применяла этот метод при $m=1$. В работе [6], где она рассматривала случай $m>1$, был использован другой метод, основанный на применении теории дифференциальных уравнений для функций со значениями в гильбертовом пространстве. Этот метод требует значительно более сильных ограничений на гладкость коэффициентов. Здесь будет показано, что метод Галеркина из [7] хорошо работает и при $m>1$ (что, впрочем, отмечается в [7]).

ДокАЗАТЕЛЬство. Пусть задача (0.2)-(0.4) имеет два обобщенных решения $u_{1}$ и $u_{2}$. Обозначим $u=u_{1}-u_{2}$. Из $(0.5)$ следует при $\eta=u_{t}$ :

$$
\begin{aligned}
(-1)^{m} \int_{\Omega_{\tau}}\left[\sum_{|p|,|q|=1}^{m}(-1)^{|p|} a_{p q} D^{q} u \overline{D^{p} u_{t}}\right. & \left.+\sum_{|p|=1}^{m} a_{p} D^{p} u \overline{u_{t}}+a u \overline{u_{t}}\right] d x d t \\
& +\int_{\Omega_{\tau}} u_{t t} \overline{u_{t}} d x d t=0, \quad 0<\tau \leqslant T .
\end{aligned}
$$

Сложив равенство (1.2) с его комплексно-сопряженным, находим

$$
\begin{aligned}
(-1)^{m} & \int_{\Omega_{\tau}} \sum_{|p|,|q|=0}^{m}(-1)^{|p|} \frac{\partial}{\partial t}\left(a_{p q} D^{q} u \overline{D^{p} u}\right) d x d t+\int_{\Omega_{\tau}} \frac{\partial}{\partial t}\left(u_{t} \overline{u_{t}}\right) d x d t \\
= & \int_{\Omega_{\tau}} \sum_{|p|,|q|=0}^{m}(-1)^{m+|p|} \frac{\partial a_{p q}}{\partial t} D^{q} u \overline{D^{p} u} d x d t \\
& -(-1)^{m} 2 \operatorname{Re} \int_{\Omega_{\tau}}\left[\sum_{|p|=1}^{m} a_{p} D^{p} u \overline{u_{t}}+a_{0} u \overline{u_{t}}\right] d x d t
\end{aligned}
$$

где $a_{00}=(-1)^{m} \lambda_{0} I, a_{0}=a-a_{00}, \lambda_{0}$ из (0.6), $I-$ единичная матрица. С помошью интегрирования по частям и неравенства $(0.6)$ получим

$$
J(\tau) \leqslant C \int_{0}^{\tau} J(t) d t
$$

где

$$
J(\tau)=\int_{\Omega}\left[\left|u_{t}(x, \tau)\right|^{2}+\sum_{|p| \leqslant m}\left|D^{p} u(x, \tau)\right|^{2}\right] d x .
$$

Отсюда и из классического неравенства Гронуолла-Беллмана следует, что $J(\tau) \equiv 0$, т.е. $u_{1}=u_{2}$ в $\Omega_{T}$.

Сушествование решения будем доказывать методом Галеркина. 
Пусть $\left\{\varphi_{k}(x)\right\}_{k=1}^{\infty}$ - система функций из $\stackrel{\circ}{H^{m}}(\Omega)$, замыкание линейной оболочки которых есть $\stackrel{\circ}{H}^{m}(\Omega)$, и эта система ортонормирована в $L_{2}(\Omega)$. Рассмотрим последовательность $u^{N}(x, t)$ вида $u^{N}(x, t)=\sum_{k=1}^{N} c_{k}^{N}(t) \varphi_{k}(x)$, где $c_{k}^{N}(t)$ таковы, что

$$
\begin{gathered}
\int_{\Omega}\left[u_{t t}^{N} \overline{\varphi_{l}}+\sum_{|p|,|q|=0}^{m}(-1)^{m+|p|} a_{p q} D^{q} u^{N} \overline{D^{p} \varphi_{l}}\right] d x \\
+(-1)^{m} \int_{\Omega}\left(\sum_{|p|=1}^{m} a_{p} D^{p} u^{N}+a_{0} u^{N}\right) \overline{\varphi_{l}} d x \\
=-\int_{\Omega} f \overline{\varphi_{l}} d x, \quad l=1, \ldots, N \\
c_{k}^{N}(0)=0, \quad \frac{d}{d t} c_{k}^{N}(0)=0 .
\end{gathered}
$$

Здесь и далее обозначаем $B_{0}(w, w)=B(w, w)+(-1)^{m} \lambda_{0}\|w\|_{L_{2}(\Omega)}^{2}$. $\mathrm{Y}_{\text {мно- }}$ жим (1.3) на $\frac{d c_{l}^{N}(t)}{d t}$ и просуммируем по $l$ от 0 до $N$. Проинтегрируем полученное равенство от 0 до $t$, после этого сложим его с комплексно-сопряженным. В результате получим

$$
\begin{aligned}
& (-1)^{m} B_{0}\left(u^{N}, u^{N}\right)(t)+\int_{\Omega}\left|u_{t}^{N}(x, t)\right|^{2} d x \\
& +(-1)^{m} 2 \operatorname{Re} \int_{\Omega_{t}}\left[\sum_{|p|=1}^{m} a_{p} D^{p} u^{N} \overline{u_{t}^{N}}+a_{0} u^{N} \overline{u_{t}^{N}}\right] d x d t \\
& -\sum_{|p|,|q|=0}^{m}(-1)^{|p|+m} \int_{\Omega_{t}} \frac{\partial a_{p q}}{\partial t} D^{q} u^{N} \overline{D^{p} u^{N}} d x d t \\
& =-2 \operatorname{Re} \int_{\Omega_{t}} f \overline{u_{t}^{N}} d x d t .
\end{aligned}
$$

Положим

$$
J_{N}(t)=\left\|u^{N}(x, t)\right\|_{H^{m}(\Omega)}^{2}+\left\|u_{t}^{N}(x, t)\right\|_{L_{2}(\Omega)}^{2} .
$$

Из (1.5) и (0.6) имеем

$$
J_{N}(t) \leqslant C_{1}\left[\int_{0}^{t} J_{N}(\tau) d \tau+\|f\|_{L_{2}\left(\Omega_{T}\right)}^{2}\right], \quad C_{1}=\text { const }
$$

Отсюда с помощью неравенства Гронуолла-Беллмана получаем

$$
\left\|u^{N}\right\|_{H^{m}\left(\Omega_{T}\right)}^{2}+\left\|u_{t}^{N}\right\|_{L_{2}\left(\Omega_{T}\right)}^{2} \leqslant C_{2}\|f\|_{L_{2}\left(\Omega_{T}\right)}^{2},
$$

где $C_{2}$ - константа, не зависящая от $N$. 
Продифференцируем каждое из соотношений (1.3) по $t$, потом умножим на $\frac{d^{2} c_{l}^{N}}{d t^{2}}$ и просуммируем все полученные равенства по $l$ от 1 до $N$. В результате получим

$$
\begin{aligned}
\int_{\Omega} u_{t t t}^{N} \overline{u_{t t}^{N}} d x & +\sum_{|p|,|q|=0}^{m} \int_{\Omega}(-1)^{m+|p|} \frac{\partial}{\partial t}\left(a_{p q} D^{q} u^{N}\right) \overline{D^{p} u_{t t}^{N}} d x \\
& +(-1)^{m} \int_{\Omega} \frac{\partial}{\partial t}\left(\sum_{|p|=1}^{m} a_{p} D^{p} u^{N}+a_{0} u^{N}\right) \overline{u_{t t}^{N}} d x=-\int_{\Omega} f_{t} \overline{u_{t t}^{N}} d x .
\end{aligned}
$$

Отсюда находим

$$
\begin{aligned}
& \int_{\Omega_{t}} \frac{\partial}{\partial t}\left(u_{t t}^{N} \overline{u_{t t}^{N}}\right) d x d t+2 \operatorname{Re} \sum_{|p|,|q|=0}^{m} \int_{\Omega_{t}}(-1)^{m+|p|} \frac{\partial}{\partial t}\left(a_{p q} D^{q} u^{N}\right) \overline{D^{p} u_{t t}^{N}} d x d t \\
& +2 \operatorname{Re}(-1)^{m} \int_{\Omega_{t}} \frac{\partial}{\partial t}\left(\sum_{|p|=1}^{m} a_{p} D^{p} u^{N}+a_{0} u^{N}\right) \overline{u_{t t}^{N}} d x d t=-2 \operatorname{Re} \int_{\Omega_{t}} f_{t} \overline{u_{t t}^{N}} d x d t
\end{aligned}
$$

Рассуждая так же, как и при выводе (1.6), можно доказать

$$
\left\|u_{t t}^{N}\right\|_{L_{2}\left(\Omega_{T}\right)}^{2} \leqslant C_{3}\left\|f_{t}\right\|_{L_{2}\left(\Omega_{T}\right)}^{2}
$$

где $C_{3}$ - константа, не зависяшая от $N$.

Из (1.6) и (1.7) получим

$$
\left\|u^{N}\right\|_{H^{m, 2}\left(\Omega_{T}\right)}^{2} \leqslant C\left[\|f\|_{L_{2}\left(\Omega_{T}\right)}^{2}+\left\|f_{t}\right\|_{L_{2}\left(\Omega_{T}\right)}^{2}\right],
$$

где $C$ - константа, не зависящая от $N$. Следовательно, из последовательности $\left\{u^{N}\right\}_{1}^{\infty}$ можно выбрать подпоследовательность, сходящуюся слабо в $H^{m, 2}\left(\Omega_{T}\right) \mathrm{k}$ некоторому элементу $u$. Нетрудно показать, что $u$ удовлетворяет тождеству $(0.5)$. Теорема доказана.

\section{§2. Гладкость обобщенного решения по временной переменной}

В этом параграфе покажем, что обобшенное решение является гладкой функцией переменной $t$, если коэффициенты системы и ее правая часть гладкие. Никаких условий на $\Omega$ при этом не требуется.

TеOрема 2.1. Пусть $a_{p q} \in C\left(\bar{\Omega}_{T}\right),|p|=|q|=m, u$

$$
\begin{aligned}
& \text { (i) } f_{t^{k}} \in L_{2}\left(\Omega_{T}\right), \quad k \leqslant h+1, \\
& \text { (ii) } f_{t^{k}}(x, 0)=0, \quad k \leqslant h, \\
& \text { (iii) }\left|\frac{\partial^{k} a_{p q}}{\partial t^{k}}\right|,\left|\frac{\partial^{k-1} a_{p}}{\partial t^{k-1}}\right|,\left|\frac{\partial^{k-1} a}{\partial t^{k-1}}\right|<\mu,
\end{aligned}
$$

$1 \leqslant|p|,|q| \leqslant m, k \leqslant h+2, \mu=\mathrm{const},(x, t) \in \bar{\Omega}_{T}$. Тогда обобщенное решение задачи (0.2)-(0.4) имеет производные по $t$ до порядка $h$ включительно из $H^{m, 2}\left(\Omega_{T}\right)$ и справедливо неравенство

$$
\left\|u_{t^{h}}\right\|_{H^{m, 2}\left(\Omega_{T}\right)}^{2} \leqslant C \sum_{k=0}^{h+1}\left\|f_{t^{k}}\right\|_{L_{2}\left(\Omega_{T}\right)}^{2}, \quad C=\text { const } .
$$


ДокАЗАТЕЛЬство. Докажем неравенство

$$
\left\|u_{t^{h}}^{N}\right\|_{H^{m, 2}\left(\Omega_{T}\right)}^{2} \leqslant C \sum_{k=0}^{h+1}\left\|f_{t^{k}}\right\|_{L_{2}\left(\Omega_{T}\right)}^{2},
$$

где $u^{N}$ - приближенное решение из доказательства теоремы 1.1, $C$ - константа, не зависяшая от $N$. Будем применять метод индукции по $h$. Из (1.8) следует, что $(2.2)$ верно для $h=0$. Допустим, что (2.2) справедливо при замене $h$ на $h-1$.

Пусть $j \leqslant h+1$. Продифференцируем каждое из соотношений (1.3) $j$ раз по $t$, затем умножим на $\frac{d^{j+1} c_{l}^{N}}{d t^{j+1}}$ и просуммируем все полученные равенства по $l$ от 1 до $N$. После этого проинтегрируем полученное равенство от 0 до $t$ и сложим его с комплексно-сопряженным. В результате получим

$$
\begin{aligned}
& \int_{\Omega_{t}} \frac{\partial}{\partial t}\left(u_{t^{j+1}}^{N} \overline{u_{t^{j+1}}^{N}}\right) d x d t \\
& +2 \operatorname{Re} \sum_{|p|,|q|=0}^{m} \int_{\Omega_{t}}(-1)^{m+|p|} \frac{\partial^{j}}{\partial t^{j}}\left(a_{p q} D^{q} u^{N}\right) \overline{D^{p} u_{t^{j+1}}^{N}} d x d t \\
& +2 \operatorname{Re}(-1)^{m} \int_{\Omega_{t}} \frac{\partial^{j}}{\partial t^{j}}\left(\sum_{|p|=1}^{m} a_{p} D^{p} u^{N}+a_{0} u^{N}\right) \overline{u_{t^{j+1}}^{N}} d x d t \\
& \quad=-2 \operatorname{Re} \int_{\Omega_{t}} f_{t^{j}} \overline{u_{t^{j+1}}^{N}} d x d t .
\end{aligned}
$$

Так как $a_{p q}=(-1)^{|p|+|q|} a_{q p}^{*}$, то

$$
\begin{aligned}
& 2 \operatorname{Re} \sum_{|p|,|q|=0}^{m}(-1)^{|p|} \frac{\partial^{j}}{\partial t^{j}}\left(a_{p q} D^{q} u^{N}\right) \overline{D^{p} u_{t^{j+1}}^{N}} \\
& =\sum_{|p|,|q|=0}^{m}(-1)^{|p|} \frac{\partial}{\partial t}\left(a_{p q} D^{q} u_{t^{j}}^{N} \overline{D^{p} u_{t^{j}}^{N}}\right)-\sum_{|p|,|q|=0}^{m}(-1)^{|p|} \frac{\partial a_{p q}}{\partial t} D^{q} u_{t^{j}}^{N} \overline{D^{p} u_{t^{j}}^{N}} \\
& \quad+2 \operatorname{Re} \sum_{|p|,|q|=0}^{m}(-1)^{|p|} \sum_{s=1}^{j}\left(\begin{array}{l}
j \\
s
\end{array}\right) \frac{\partial^{s} a_{p q}}{\partial t^{s}} D^{q} u_{t^{j-s}}^{N} \overline{D^{p} u_{t^{j+1}}^{N}}
\end{aligned}
$$

Из (2.3), (2.4) с помощью интегрирования по частям получаем

$$
\begin{aligned}
& \left\|u_{t^{j+1}}^{N}(x, t)\right\|_{L_{2}(\Omega)}^{2}+(-1)^{m} B_{0}\left(u_{t^{j}}^{N}, u_{t^{j}}^{N}\right)(t) \\
& =\sum_{|p|,|q|=0}^{m}(-1)^{m+|p|} \int_{\Omega_{t}} \frac{\partial a_{p q}}{\partial t} D^{q} u_{t^{j}}^{N} \overline{D^{p} u_{t^{j}}^{N}} d x d t \\
& \quad-2 \operatorname{Re} \sum_{|p|,|q|=0}^{m} \sum_{s=1}^{j}(-1)^{m+|p|}\left(\begin{array}{l}
j \\
s
\end{array}\right) \int_{\Omega_{t}} \frac{\partial^{s} a_{p q}}{\partial t^{s}} D^{q} u_{t^{j-s}}^{N} \overline{D^{p} u_{t^{j+1}}^{N}} d x d t \\
& \quad-2 \operatorname{Re}(-1)^{m} \int_{\Omega_{t}} \frac{\partial^{j}}{\partial t^{j}}\left(\sum_{|p|=1}^{m} a_{p} D^{p} u^{N}+a_{0} u^{N}\right) \overline{u_{t^{j+1}}^{N}} d x d t \\
& \quad-2 \operatorname{Re} \int_{\Omega_{t}} f_{t^{j}} \overline{u_{t^{j+1}}^{N}} d x d t .
\end{aligned}
$$


Легко видеть, что

$$
\begin{aligned}
& \int_{\Omega_{t}} \frac{\partial^{s} a_{p q}}{\partial t^{s}} D^{q} u_{t^{j-s}}^{N} \overline{D^{p} u_{t^{j+1}}^{N}} d x d t=\int_{\Omega} \frac{\partial^{s} a_{p q}}{\partial t^{s}} D^{q} u_{t^{j-s}}^{N} \overline{D^{p} u_{t^{j}}^{N}} d x \\
& \quad-\int_{\Omega_{t}} \frac{\partial^{s+1} a_{p q}}{\partial t^{s+1}} D^{q} u_{t^{j-s}}^{N} \overline{D^{p} u_{t^{j}}^{N}} d x d t-\int_{\Omega_{t}} \frac{\partial^{s} a_{p q}}{\partial t^{s}} D^{q} u_{t^{j-s+1}}^{N} \overline{D^{p} u_{t^{j}}^{N}} d x d t .
\end{aligned}
$$

Используя неравенство Коши и (0.6), а также соотношения (2.5), (2.6), находим

$$
\begin{aligned}
& \left\|u_{t^{j+1}}^{N}\right\|_{L_{2}(\Omega)}^{2}+\left\|u_{t^{j}}^{N}\right\|_{H^{m}(\Omega)}^{2} \leqslant C_{1} \int_{0}^{t}\left[\left\|u_{t^{j}}^{N}\right\|_{H^{m}(\Omega)}^{2}+\left\|u_{t^{j+1}}^{N}\right\|_{L_{2}(\Omega)}^{2}\right] d t \\
& +C_{2}\left[\sum_{s=0}^{j-1}\left\|u_{t^{s}}^{N}\right\|_{H^{m}(\Omega)}^{2}+\sum_{s=0}^{j-1}\left\|u_{t^{s}}^{N}\right\|_{H^{m, 0}\left(\Omega_{T}\right)}^{2}+\left\|f_{t^{j}}\right\|_{L_{2}\left(\Omega_{T}\right)}^{2}\right],
\end{aligned}
$$

где $C_{i}=$ const $>0, i=1,2$.

Положим

$$
\begin{gathered}
y(t)=\int_{0}^{t}\left[\left\|u_{t^{j+1}}^{N}\right\|_{L_{2}(\Omega)}^{2}+\left\|u_{t^{j}}^{N}\right\|_{H^{m}(\Omega)}^{2}\right] d t \\
c_{2}(t)=C_{2}\left[\sum_{s=0}^{j-1}\left\|u_{t^{s}}^{N}\right\|_{H^{m}(\Omega)}^{2}+\sum_{s=0}^{j-1}\left\|u_{t^{s}}^{N}\right\|_{H^{m, 0}\left(\Omega_{T}\right)}^{2}+\left\|f_{t^{j}}\right\|_{L_{2}\left(\Omega_{T}\right)}^{2}\right] .
\end{gathered}
$$

Отсюда и из (2.7) следует, что

$$
\frac{d y(t)}{d t} \leqslant C_{1} y(t)+c_{2}(t)
$$

С помощью [7; гл. III, лемма 1.1] из (2.8) получим

$$
y(t) \leqslant e^{t C_{1}} \int_{0}^{t} c_{2}(t) d t .
$$

При $t=T$ будем иметь

$$
\left\|u_{t^{j}}^{N}\right\|_{H^{m, 1}\left(\Omega_{T}\right)}^{2} \leqslant C\left[\sum_{s=0}^{j-1}\left\|u_{t^{s}}^{N}\right\|_{H^{m, 0}\left(\Omega_{T}\right)}^{2}+\left\|f_{t^{j}}\right\|_{L_{2}\left(\Omega_{T}\right)}^{2}\right]
$$

где $C$ - константа, не зависяшая от $N$.

Положив $j=h$ в неравенстве $(2.9)$, получим

$$
\left\|u_{t^{h}}^{N}\right\|_{H^{m, 1}\left(\Omega_{T}\right)}^{2} \leqslant C\left[\sum_{s=0}^{h-1}\left\|u_{t^{s}}^{N}\right\|_{H^{m, 0}\left(\Omega_{T}\right)}^{2}+\left\|f_{t^{h}}\right\|_{L_{2}\left(\Omega_{T}\right)}^{2}\right] .
$$

С другой стороны, из (2.9) при $j=h+1$ следует, что

$$
\left\|u_{t^{h+2}}^{N}\right\|_{L_{2}\left(\Omega_{T}\right)}^{2} \leqslant C\left[\sum_{s=0}^{h}\left\|u_{t^{s}}^{N}\right\|_{H^{m, 0}\left(\Omega_{T}\right)}^{2}+\left\|f_{t^{h+1}}\right\|_{L_{2}\left(\Omega_{T}\right)}^{2}\right] .
$$

Следовательно,

$$
\left\|u_{t^{h}}^{N}\right\|_{H^{m, 2}\left(\Omega_{T}\right)}^{2} \leqslant C\left[\sum_{s=0}^{h-1}\left\|u_{t^{s}}^{N}\right\|_{H^{m, 0}\left(\Omega_{T}\right)}^{2}+\left\|f_{t^{h}}\right\|_{L_{2}\left(\Omega_{T}\right)}^{2}+\left\|f_{t^{h+1}}\right\|_{L_{2}\left(\Omega_{T}\right)}^{2}\right]
$$

где $C$ - константа, не зависящая от $N$.

Неравенство (2.2) следует из (2.10) и индуктивной гипотезы. Оценка (2.2) позволяет вьполнить предельный переход по $N \rightarrow \infty$. Теорема 2.1 доказана. 


\section{§ 3. Гладкость обобщенного решения в областях с конической точкой на границе}

В этом и следуюшем параграфе предполагаем, что коэффициенты оператора $L$ - бесконечно дифференцируемые функции в $\bar{\Omega}_{T}$. Граница $\partial \Omega$ области $\Omega$ предполагается бесконечно гладкой вне начала координат, а в малой окрестности начала координат она является конической поверхностью, т.е. существует такое $\delta>0$, что $\{x: x \in \Omega,|x|<\delta\}=\{x: x \in K,|x|<\delta\}$, где $K-$ конус в $\mathbb{R}^{n}$, т.е. $K=\{x: x /|x| \in G\}$, где $G$ - гладкая область на единичной сфере $S^{n-1}$.

ЛЕмма 3.1. Если $f, f_{t} \in L_{2}\left(K_{T}\right), f(x, 0)=0 u и$ - обобщенное решение задачи (0.2)-(0.4) такое, что $u \equiv 0$ при $|x|>R=$ const, mо $u \in H_{m}^{2 m, 2}\left(K_{T}\right) u$

$$
\|u\|_{H_{m}^{2 m, 2}\left(K_{T}\right)}^{2} \leqslant C\left[\|f\|_{L_{2}\left(K_{T}\right)}^{2}+\left\|f_{t}\right\|_{L_{2}\left(K_{T}\right)}^{2}\right], \quad C=\text { const } .
$$

ДокАЗАТЕльство. Перепишем систему (0.2) в виде

$$
\sum_{|\alpha| \leqslant 2 m} b_{\alpha}(x, t) D^{\alpha} u=F
$$

где $u_{t t}+f=F$. Из теоремы 2.1 следует, что $F \in L_{2}\left(K_{T}\right)$.

Рассмотрим последовательность областей $\Omega_{k}$,

$$
\Omega_{k}=\left\{x: x \in K, 2^{-k} \leqslant|x| \leqslant 2^{-k+1}\right\}, \quad k=1,2, \ldots
$$

Из теорем о гладкости решения эллиптических систем внутри области и вблизи гладкого куска гранищы [11] следует, что

$$
\begin{gathered}
\int_{\Omega_{2}}\left|D^{\alpha} u(x, t)\right|^{2} d x \leqslant C \int_{\Omega_{1} \cup \Omega_{2} \cup \Omega_{3}}\left(|F(x, t)|^{2}+|u(x, t)|^{2}\right) d x \\
|\alpha| \leqslant 2 m, \quad C=\text { const } .
\end{gathered}
$$

Сделаем в $(3.1)$ замену $x=\left(4 / 2^{k_{1}}\right) x^{\prime}$ при $k_{1}>2$. Затем применим оценку $(3.2)$. В результате получим

$$
\begin{gathered}
\int_{\Omega_{2}}\left|D_{x^{\prime}}^{\alpha} u\left(x^{\prime}, t\right)\right|^{2} d x^{\prime} \leqslant C_{1} \int_{\Omega_{1} \cup \Omega_{2} \cup \Omega_{3}}\left(\left|F\left(x^{\prime}, t\right)\right|^{2}\left(\frac{4}{2^{k_{1}}}\right)^{4 m}+\left|u\left(x^{\prime}, t\right)\right|^{2}\right) d x^{\prime} \\
C_{1}=\text { const }
\end{gathered}
$$

Возвращаясь к переменным $x_{1}, \ldots, x_{n}$, находим:

$$
\begin{aligned}
& \int_{\Omega_{k_{1}}}\left|D^{\alpha} u(x, t)\right|^{2} r^{2(|\alpha|-m)} d x \\
& \quad \leqslant C_{2} \int_{\Omega_{k_{1}-1} \cup \Omega_{k_{1}} \cup \Omega_{k_{1}+1}}\left(|F(x, t)|^{2} r^{2 m}+r^{-2 m}|u(x, t)|^{2}\right) d x, \quad C_{2}=\text { const . }
\end{aligned}
$$


Отсюда получим

$$
\int_{K}\left|D^{\alpha} u(x, t)\right|^{2} r^{2(|\alpha|-m)} d x \leqslant C_{2} \int_{K}\left(|F(x, t)|^{2} r^{2 m}+r^{-2 m}|u(x, t)|^{2}\right) d x .
$$

Из условий $\left.D_{\nu}^{j} u\right|_{S_{T}}=0, j=0,1, \ldots, m-1$, следует оценка

$$
\int_{K} r^{-2 m}|u(x, t)|^{2} d x \leqslant C_{3} \int_{K}\left|D^{m} u\right|^{2} d x, \quad C_{3}=\text { const } .
$$

Из (3.3) и (3.4) имеем:

$$
\int_{K} r^{2(|\alpha|-m)}\left|D^{\alpha} u(x, t)\right|^{2} d x \leqslant C_{4} \int_{K}\left(|f|^{2}+\left|u_{t t}\right|^{2}+\left|D^{m} u\right|^{2}\right) d x, \quad C_{4}=\text { const } .
$$

Проинтегрировав это неравенство по $t$ от 0 до $T$, получим

$$
\int_{K_{T}} r^{2(|\alpha|-m)}\left|D^{\alpha} u(x, t)\right|^{2} d x d t \leqslant C_{4} \int_{K_{T}}\left(|f|^{2}+\left|u_{t t}\right|^{2}+\left|D^{m} u\right|^{2}\right) d x d t .
$$

В силу теоремы 1.1 из (3.5) следует утверждение леммы. Лемма доказана.

Пусть $\omega$ - локальная система координат на $S^{n-1}$. Главную часть оператора $L$ в точке 0 можно записать в виде

$$
L_{0}(0, t ; D)=r^{-2 m} Q\left(\omega, t, r D_{r}, D_{\omega}\right), \quad D_{r}=i \frac{\partial}{\partial r},
$$

где $Q$ - линейный оператор с гладкими коэффициентами.

В дальнейшем большое значение будет иметь спектральная задача:

$$
\begin{gathered}
Q\left(\omega, t, \lambda, D_{\omega}\right) v(\omega)=0, \quad \omega \in G, \\
D_{\omega}^{j} v(\omega)=0, \quad \omega \in \partial G, \quad j=0, \ldots, m-1 .
\end{gathered}
$$

Известно, что при каждом $t \in[0, T]$ спектр ее дискретньй.

Докажем следующую теорему.

ТеОРема 3.1. Пусть $и$ - обобщенное решение задачи (0.2)-(0.4), $|x|>R=$ const, u nycms $f_{t^{k}} \in L_{2}\left(K_{T}\right)$ npu $k \leqslant 2 m, f_{t^{k}}(x, 0)=0$ npu $k \leqslant 2 m-1$. Кроме того, предположим, что полоса

$$
m-\frac{n}{2} \leqslant \operatorname{Im} \lambda \leqslant 2 m-\frac{n}{2}
$$

не содержит точек спектра задачи (3.6)-(3.7) ни при каких $t \in[0, T]$. Тогда $u \in H^{2 m}\left(K_{T}\right)$ и справедливо неравенство

$$
\|u\|_{H^{2 m}\left(K_{T}\right)}^{2} \leqslant C \sum_{k \leqslant 2 m}\left\|f_{t^{k}}\right\|_{L_{2}\left(K_{T}\right)}^{2}, \quad C=\text { const. }
$$


ДокАЗАТЕЛЬство. Сначала докажем следуюшее неравенство.

$$
\left\|u_{t^{s}}\right\|_{H_{0}^{2 m, 0}\left(K_{T}\right)}^{2} \leqslant C \sum_{k \leqslant 2 m}\left\|f_{t^{k}}\right\|_{L_{2}\left(K_{T}\right)}^{2}, \quad C=\mathrm{const}, \quad s \leqslant 2 m-1 .
$$

Из (0.2) следует, что

$$
(-1)^{m-1} L_{0}(0, t ; D) u=F(x, t),
$$

где $F(x, t)=u_{t t}+f+(-1)^{m-1}\left[L_{0}(0, t ; D)-L(x, t ; D)\right] u$. Из теоремы 1.1 и леммы 3.1 следует, что $F \in H_{m-1}^{0,0}\left(K_{T}\right)$. Так как в полосе $m-n / 2 \leqslant \operatorname{Im} \lambda \leqslant m+1-n / 2$ нет точек спектра задачи (3.6)-(3.7) ни при каких $t \in[0, T]$, то из теоремы 3.2 работы [12] и априорной оценки решений эллиптических систем [13] получим, что $u \in H_{m-1}^{2 m}(K)$ при почти всех $t \in[0, T]$ и

$$
\begin{aligned}
\|u\|_{H_{m-1}^{2 m}(K)}^{2} & \leqslant C\left[\|F\|_{H_{m-1}^{0}(K)}^{2}+\|u\|_{H_{m}^{2 m}(K)}^{2}\right] \\
& \leqslant C\left[\|f\|_{L_{2}(K)}^{2}+\left\|u_{t t}\right\|_{L_{2}(K)}^{2}+\|u\|_{H_{m}^{2 m}(K)}^{2}\right],
\end{aligned}
$$

где $C=$ const. Повторение проведенных вьше рассуждений приводит к неравенству

$$
\|u\|_{H_{0}^{2 m}(K)}^{2} \leqslant C\left[\|f\|_{L_{2}(K)}^{2}+\left\|u_{t t}\right\|_{L_{2}(K)}^{2}+\|u\|_{H_{m}^{2 m}(K)}^{2}\right]
$$

и, более того,

$$
\|u\|_{H_{0}^{2 m, 0}\left(K_{T}\right)}^{2} \leqslant C\left[\|f\|_{L_{2}\left(K_{T}\right)}^{2}+\|u\|_{H_{m}^{2 m, 2}\left(K_{T}\right)}^{2}\right] .
$$

Отсюда и из леммы 3.1 следует, что

$$
\|u\|_{H_{0}^{2 m, 0}\left(K_{T}\right)}^{2} \leqslant C\left[\|f\|_{L_{2}\left(K_{T}\right)}^{2}+\left\|f_{t}\right\|_{L_{2}\left(K_{T}\right)}^{2}\right],
$$

т.е. (3.8) доказано при $s=0$.

Пусть (3.8) верно для $s-1$. Продифференцируем систему (0.2) $s$ раз по $t$ и положим $v=u_{t} s$. В результате получим

$$
(-1)^{m-1} L v=v_{t t}+f_{t^{s}}+\sum_{k=1}^{s}\left(\begin{array}{l}
s \\
k
\end{array}\right) L_{k} u_{t^{s-k}},
$$

где

$$
L_{k}=\sum_{|p|,|q|=1}^{m} D^{p} \frac{\partial^{k} a_{p q}}{\partial t^{k}} D^{q}+\sum_{|p|=1}^{m} \frac{\partial^{k} a_{p}}{\partial t^{k}} D^{p}+\frac{\partial^{k} a}{\partial t^{k}} .
$$

Из предположения индукции, рассуждая так же, как в случае $s=0$, получим (3.8).

Так как

$$
\|u\|_{H^{2 m}\left(K_{T}\right)}^{2} \leqslant \sum_{s \leqslant 2 m-1}\left\|u_{t^{s}}\right\|_{H_{0}^{2 m, 0}\left(K_{T}\right)}^{2}+\left\|u_{t^{2 m}}\right\|_{L_{2}\left(K_{T}\right)}^{2},
$$

то из (3.8) и теоремы 2.1 следует утверждение теоремы. Теорема доказана. 
Лемма 3.2. Пусть и - обобщенное решение задачи (0.2)-(0.4) такое, что $u \equiv 0$ nрu $|x|>R=$ const. Пусть, кроме того, $u \in H_{\beta-1}^{2 m+l-1,0}\left(K_{T}\right), u_{t t} \in$ $H_{\beta}^{l, 0}\left(K_{T}\right), f \in H_{\beta}^{l, 0}\left(K_{T}\right)$. Тогда $u \in H_{\beta}^{2 m+l, 0}\left(K_{T}\right) u$

$$
\|u\|_{H_{\beta}^{2 m+l, 0}\left(K_{T}\right)}^{2} \leqslant C\left[\|f\|_{H_{\beta}^{l, 0}\left(K_{T}\right)}^{2}+\left\|u_{t t}\right\|_{H_{\beta}^{l, 0}\left(K_{T}\right)}^{2}+\|u\|_{H_{\beta-1}^{2 m+l-1,2}\left(K_{T}\right)}^{2}\right],
$$

əде $C=$ const.

ДокАЗАТЕЛьСтво. Известно [11], что

$$
\int_{\Omega_{2}}\left|D^{2 m+l} u(x, t)\right|^{2} d x \leqslant C \int_{\Omega_{1} \cup \Omega_{2} \cup \Omega_{3}}\left(\sum_{|\alpha| \leqslant l}\left|D^{\alpha} F(x, t)\right|^{2}+|u(x, t)|^{2}\right) d x,
$$

где $F=u_{t t}+f, C=$ const. Из этого неравенства, рассуждая, как при доказательстве (3.3), получаем

$$
\begin{aligned}
& \int_{K} r^{2 \beta}\left|D^{2 m+l} u(x, t)\right|^{2} d x \\
& \quad \leqslant C \int_{K}\left(\sum_{|\alpha| \leqslant l} r^{2(\beta+|\alpha|-l)}\left|D^{\alpha} F(x, t)\right|^{2}+r^{2(\beta-2 m-l)}|u(x, t)|^{2}\right) d x .
\end{aligned}
$$

Интегрируя это равенство по $t$ от 0 до $T$, находим:

$$
\begin{aligned}
& \int_{K_{T}} r^{2 \beta}\left|D^{2 m+l} u(x, t)\right|^{2} d x d t \\
& \quad \leqslant C\left[\|F\|_{H_{\beta}^{l, 0}\left(K_{T}\right)}^{2}+\int_{K_{T}} r^{2(\beta-2 m-l)}|u(x, t)|^{2} d x d t\right] \\
& \quad \leqslant C\left[\|F\|_{H_{\beta}^{l, 0}\left(K_{T}\right)}^{2}+\|u\|_{H_{\beta-1}^{2 m+l-1,0}\left(K_{T}\right)}^{2}\right] .
\end{aligned}
$$

Имеем:

$$
\|u\|_{H_{\beta}^{2 m+l, 0}\left(K_{T}\right)}^{2}=\int_{K_{T}} r^{2 \beta}\left|D^{2 m+l} u(x, t)\right|^{2} d x d t+\|u\|_{H_{\beta-1}^{2 m+l-1,0}\left(K_{T}\right)}^{2} .
$$

Отсюда и из (3.12) следует утверждение леммы. Лемма доказана.

Tеорема 3.2. Пусть $f_{t^{k}} \in H^{l}\left(K_{T}\right) n p u k \leqslant 2 m, f_{t^{k}}(x, 0)=0 n p u k \leqslant l+$ $2 m-1$, и пусть и - обобщенное решение задачи (0.2)-(0.4) такое, что и 0 при $|x|>R=$ const. Кроме того, предположсим, что в полосе

$$
m-\frac{n}{2} \leqslant \operatorname{Im} \lambda \leqslant 2 m+l-\frac{n}{2}
$$

нет точек спектра задачи (3.6)-(3.7) ни при каких $t \in[0, T]$. Тогда и $\in$ $H^{2 m+l}\left(K_{T}\right)$ и справедливо неравенство

$$
\|u\|_{H^{2 m+l}\left(K_{T}\right)}^{2} \leqslant C \sum_{k=0}^{2 m}\left\|f_{t^{k}}\right\|_{H^{l}\left(K_{T}\right)}^{2}, \quad C=\text { const }
$$


ДокАЗАТЕльство. Теорема будет доказываться индукцией по $l$. При $l=0$ утверждение теоремы следует из теоремы 3.1. Пусть утверждение теоремы верно при замене $l$ на $l-1$.

Докажем неравенство:

$$
\left\|u_{t^{s}}\right\|_{H^{2 m+l-s}\left(K_{T}\right)}^{2} \leqslant C \sum_{k=0}^{2 m-1}\left\|f_{t^{k}}\right\|_{H^{l}\left(K_{T}\right)}^{2}
$$

при $s=l, l-1, \ldots, 0$, где $C=$ const.

Рассуждая так же, как и при доказательстве теоремы 3.1 , можно получить неравенство (3.13) для $s=l$. Допустим, что (3.13) верно при $s=l, l-1, \ldots, j+1$.

Положим $v=u_{t j}$. Из (3.10) следует

$$
(-1)^{m-1} L v=F_{j},
$$

где

$$
F_{j}=v_{t t}+f_{t^{j}}+\sum_{k=1}^{j}\left(\begin{array}{l}
j \\
k
\end{array}\right) L_{k} u_{t^{j-k}} .
$$

Из индуктивной гипотезы по $l$ получим

$$
\sum_{k=1}^{j}\left(\begin{array}{l}
j \\
k
\end{array}\right) L_{k} u_{t^{j-k}} \in H^{l-j}\left(K_{T}\right) .
$$

С другой стороны, с помощью предположения индукции по $s$ имеем $v_{t t} \in H^{l-j}\left(K_{T}\right)$. Следовательно, $F_{j} \in H^{l-j}\left(K_{T}\right)$. Так как $H_{-1}^{l-j-1,0}\left(K_{T}\right) \in H^{l-j}\left(K_{T}\right)$, то $F_{j} \in$ $H_{-1}^{l-j-1,0}\left(K_{T}\right)$.

Повторяя рассуждения, аналогичные доказательству теоремы 3.1 , получаем: $v \in H_{-1}^{2 m+l-j-1,0}\left(K_{T}\right)$. Отсюда и из леммы 3.2 имеем $u_{t^{j}}=v \in H_{0}^{2 m+l-j, 0}\left(K_{T}\right)$ И

$$
\left\|u_{t^{j}}\right\|_{H_{0}^{2 m+l-j, 0}\left(K_{T}\right)}^{2} \leqslant C \sum_{k=0}^{2 m-1}\left\|f_{t^{k}}\right\|_{H^{l}\left(K_{T}\right)}^{2}, \quad C=\text { const } .
$$

Тогда

$$
\left\|u_{t^{j}}\right\|_{H^{2 m+l-j}\left(K_{T}\right)}^{2}=\left\|u_{t^{j+1}}\right\|_{H^{2 m+l-j-1}\left(K_{T}\right)}^{2}+\left\|u_{t^{j}}\right\|_{H_{0}^{2 m+l-j, 0}\left(K_{T}\right)}^{2} .
$$

Из (3.13) при $s=j+1$ получим

$$
\left\|u_{t^{j+1}}\right\|_{H^{2 m+l-j-1}\left(K_{T}\right)}^{2} \leqslant C \sum_{k=0}^{2 m-1}\left\|f_{t^{k}}\right\|_{H^{l}\left(K_{T}\right)}^{2}, \quad C=\mathrm{const} .
$$

Отсюда и из $(3.15),(3.16)$ следует, что

$$
\left\|u_{t^{j}}\right\|_{H^{2 m+l-j}\left(K_{T}\right)}^{2} \leqslant C \sum_{k=0}^{2 m-1}\left\|f_{t^{k}}\right\|_{H^{l}\left(K_{T}\right)}^{2}, \quad C=\text { const } .
$$

При $j=0$ получим утверждение теоремы.

Переходим теперь к теореме о гладкости обобщенного решения в областях с конической точкой на границе. 
ТЕОРемА 3.3. Пусть и - обобщенное решение задачи (0.2)-(0.4), и пусть $f_{t^{k}} \in H^{l}\left(\Omega_{T}\right)$ nри $k \leqslant 2 m, f_{t^{k}}(x, 0)=0$ при $k \leqslant l+2 m-1$. Кроме того, предположим, что полоса

$$
m-\frac{n}{2} \leqslant \operatorname{Im} \lambda \leqslant l+2 m-\frac{n}{2}
$$

не содержит точек спектра задачи (3.6)-(3.7) ни при каких $t \in[0, T]$. Тогда $u \in H^{2 m+l}\left(\Omega_{T}\right)$ и справедливо неравенство

$$
\|u\|_{H^{2 m+l}\left(\Omega_{T}\right)}^{2} \leqslant C \sum_{k=0}^{2 m-1}\left\|f_{t^{k}}\right\|_{H^{l}\left(\Omega_{T}\right)}^{2}, \quad C=\mathrm{const} .
$$

ДокАЗАТЕЛЬСТво. Точку 0 окружим окрестностью $U_{0}$ столь малого диаметpa, что пересечение $\Omega$ и $U_{0}$ совпадает с $K$.

Рассмотрим функцию $\varphi_{0} \in \stackrel{\circ}{C^{\infty}}\left(U_{0}\right)$, равную единице в некоторой окрестности нуля. Она удовлетворяет системе

$$
(-1)^{m-1} L(x, t ; D) u_{0}-\left(u_{0}\right)_{t t}=\varphi_{0} f+L^{*}(x, t ; D) u_{0}, \quad u_{0}=\varphi_{0} u,
$$

где $L^{*}$ - линейный дифференциальный оператор порядка менше чем $2 m$. Коэффициенты этого оператора зависят от выбора функции $\varphi_{0}$ и обращаются в нуль вне $U_{0}$. Отсюда и из рассуждений, аналогичных доказательству теоремы 3.2 , получим

$$
\left\|\varphi_{0} u\right\|_{H^{2 m+l}\left(\Omega_{T}\right)}^{2} \leqslant C \sum_{k=0}^{2 m-1}\left\|f_{t^{k}}\right\|_{H^{l}\left(\Omega_{T}\right)}^{2} .
$$

Функция $\varphi_{1} u=\left(1-\varphi_{0}\right) u$ обращается в нуль в окрестности конической точки. $\mathrm{K} \varphi_{1} u$ можно применить теоремы о дифференцируемости решения эллиптической задачи в гладкой области, на основании которых заключаем, что $\varphi_{1} u \in H^{2 m+l}(\Omega)$ и, тем более, $\varphi_{1} u \in H^{2 m+l}\left(\Omega_{T}\right)$, при этом

$$
\left\|\varphi_{1} u\right\|_{H^{2 m+l}\left(\Omega_{T}\right)}^{2} \leqslant C \sum_{k=0}^{2 m-1}\left\|f_{t^{k}}\right\|_{H^{l}\left(\Omega_{T}\right)}^{2} .
$$

Представим теперь функцию $u(x, t)$ в виде

$$
u(x, t)=\varphi_{0} u(x, t)+\varphi_{1} u(x, t) .
$$

Отсюда и из (3.17), (3.18) получим

$$
\|u\|_{H^{2 m+l}\left(\Omega_{T}\right)}^{2} \leqslant C \sum_{k=0}^{2 m-1}\left\|f_{t^{k}}\right\|_{H^{l}\left(\Omega_{T}\right)}^{2}, \quad C=\text { const } .
$$

Теорема доказана. 


\section{§4. Асимптотика решения в окрестности конической точки}

В этом параграфе установим асимптотические формулы для обобщенного решения задачи $(0.2)-(0.4)$ в области $\Omega$, определенной в $\S 3$.

Сначала рассмотрим в $K$ задачу Дирихле для системы:

$$
L_{0} u=r^{-i \lambda_{0}(t)-2 m} \sum_{s=0}^{M} \ln ^{s} r f_{s}(\omega, t) .
$$

Справедлива следуюшая лемма [13].

ЛЕмма 4.1. Пусть $f_{s}(\omega, t), s=0, \ldots, M,-$ бесконечно дифферениируемые по ш функиии. Тогда существует решение системы (4.1) вида

$$
u(x, t)=r^{-i \lambda_{0}} \sum_{s=0}^{M+\nu} \ln ^{s} r \tilde{f}_{s}(\omega, t)
$$

где $\tilde{f}_{s}, s=0, \ldots, M+\nu,-$ бесконечно дифференцируемые по $\omega$ функции, $\nu=1$, если $\lambda_{0}$ - простое собственное значение задачи (3.6)-(3.7), и $\nu=0$, если $\lambda_{0}$ не является собственным значением задачи (3.6)-(3.7).

ЛЕмма 4.2. Пусть $и$ - решение задачи (0.2)-(0.4) такое, что и 0 при $|x|>R=$ const, u nycmb $u_{t^{k}} \in H_{\beta}^{2 m+l, 0}\left(K_{T}\right), u_{t^{k+2}} \in H_{\beta^{\prime}}^{l, 0}\left(K_{T}\right), f_{t^{k}} \in H_{\beta^{\prime}}^{l, 0}\left(K_{T}\right)$, $k \leqslant h, \beta>\beta^{\prime}$. Кроме того, предположсим, что прямые

$$
\operatorname{Im} \lambda=-\beta+2 m+l-\frac{n}{2} \quad u \quad \operatorname{Im} \lambda=-\beta^{\prime}+2 m+l-\frac{n}{2}
$$

не содержат точек спектра задачи (3.6)-(3.7) ни при каких $t \in[0, T]$, а в nолосе

$$
-\beta+2 m+l-\frac{n}{2}<\operatorname{Im} \lambda<-\beta^{\prime}+2 m+l-\frac{n}{2}
$$

есть только одно простое собственное значение $\lambda(t)$ задачи (3.6)-(3.7). Тогда справедливо представление

$$
u(x, t)=c(t) r^{-i \lambda(t)} \varphi(\omega, t)+u_{1}(x, t),
$$

где аргументов, $c_{t^{k}} \in L_{2}(0, T) u\left(u_{1}\right)_{t^{k}} \in H_{\beta^{\prime}}^{2 m+l, 0}\left(K_{T}\right) n p u k \leqslant h$.

ДокАЗАТЕЛЬСтво. Из [12; теорема 3.2$]$ следует, что

$$
u(x, t)=c(t) r^{-i \lambda(t)} \varphi(\omega, t)+u_{1}(x, t),
$$

где $\varphi(\omega, t)-$ собственная функция задачи (3.6)-(3.7), отвечающая собственному значению $\lambda(t), u_{1} \in H_{\beta^{\prime}}^{2 m+l}(K)$ и $c(t)$ определяется формулой:

$$
c(t)=i \int_{K} F(x, t) r^{-i \overline{\lambda(t)}+2 m-n} \psi(x, t) d x,
$$


где $F=u_{t t}+f, \psi$-собственная функция задачи, сопряженной к задаче (3.6)-(3.7), соответствуюшая собственному значению $\overline{\lambda(t)}$. Так как $\operatorname{Im} \overline{\lambda(t)}>-\beta^{\prime}-2 m-l+$ $n / 2$ и $F \in H_{\beta^{\prime}}^{l, 0}\left(K_{T}\right)$, то $c(t) \in L_{2}(0, T)$. Лемма доказана при $h=0$.

Допустим, что утверждение леммы справедливо для $h-1$. Продифференцируем систему (3.9) $h$ раз по $t$ и положим $v=u_{t}$. В результате получим

$$
(-1)^{m-1} L_{0} v=F_{t^{h}}+(-1)^{m} \sum_{k=1}^{h}\left(\begin{array}{l}
h \\
k
\end{array}\right) L_{0 t^{k}} u_{t^{h-k}}
$$

где $L_{0 t^{k}}=\sum_{|p|=|q|=m} \frac{\partial^{k} a_{p q}(0, t)}{\partial t^{k}} D^{p} D^{q}$.

Положим $S_{0}(\omega, t)=r^{-i \lambda(t)} \varphi_{0}(\omega, t)$. Так как $\varphi_{0}(\omega, t) \in C^{\infty}(\omega, t)[14]$, то из $(4.4)$ находим

$$
\sum_{k=1}^{h}\left(\begin{array}{l}
h \\
k
\end{array}\right) L_{0 t^{k}} u_{t^{h-k}}=\sum_{k=1}^{h}\left(\begin{array}{l}
h \\
k
\end{array}\right) L_{0 t^{k}}\left[c\left(S_{0}\right)_{t^{h-k}}\right]+\sum_{k=1}^{h}\left(\begin{array}{l}
h \\
k
\end{array}\right) L_{0 t^{k}}\left(u_{1}\right)_{t^{h-k}}
$$

Из этого равенства с помощью индуктивной гипотезы получаем

$$
\sum_{k=1}^{h}\left(\begin{array}{l}
h \\
k
\end{array}\right) L_{0 t^{k}} u_{t^{h-k}}=F_{1}-\sum_{k=1}^{h}\left(\begin{array}{l}
h \\
k
\end{array}\right) c_{t^{h-k}} L_{0}\left(S_{0}\right)_{t^{k}}
$$

где $F_{1} \in H_{\beta^{\prime}}^{l, 0}\left(K_{T}\right)$. Из (4.4) и (4.5) имеем

$$
(-1)^{m-1} L_{0} v=F_{2}-\sum_{k=1}^{h}\left(\begin{array}{l}
h \\
k
\end{array}\right) c_{t^{h-k}} L_{0}\left(S_{0}\right)_{t^{k}}
$$

где $F_{2} \in H_{\beta^{\prime}}^{l, 0}\left(K_{T}\right)$. Отсюда и из леммы 4.1 находим

$$
u_{t^{h}}=v=\sum_{k=1}^{h}\left(\begin{array}{l}
h \\
k
\end{array}\right) c_{t^{h-k}}\left(S_{0}\right)_{t^{k}}+d(t) S_{0}+u_{2}
$$

где $d(t) \in L_{2}(0, T), u_{2} \in H_{\beta^{\prime}}^{l, 0}\left(K_{T}\right)$. Из этого равенства следует, что

$$
S_{0,1}=u_{t^{h}}-\sum_{k=2}^{h}\left(\begin{array}{l}
h \\
k
\end{array}\right) c_{t^{h-k}}\left(S_{0}\right)_{t^{k}}-(h-1) c_{t^{h-1}}\left(S_{0}\right)_{t}=c_{t^{h-1}}\left(S_{0}\right)_{t}+d S_{0}+u_{2}
$$

Теперь продифференцируем равенство (4.3) $h-1$ раз по $t$. В результате получим

$$
u_{t^{h-1}}=\sum_{k=0}^{h-1}\left(\begin{array}{c}
h-1 \\
k
\end{array}\right) c_{t^{h-k-1}}\left(S_{0}\right)_{t^{k}}+\left(u_{1}\right)_{t^{h-1}}
$$


Перепишем (4.9) в виде

$$
S_{0,2}=u_{t^{h-1}}-\sum_{k=1}^{h-1}\left(\begin{array}{c}
h-1 \\
k
\end{array}\right) c_{t^{h-k-1}}\left(S_{0}\right)_{t^{k}}=c_{t^{h-1}} S_{0}+\left(u_{1}\right)_{t^{h-1}}
$$

Следовательно,

$$
\begin{aligned}
\left(S_{0,2}\right)_{t} & =u_{t^{h}}-\sum_{k=1}^{h-1}\left(\begin{array}{c}
h-1 \\
k
\end{array}\right)\left[c_{t^{h-k}}\left(S_{0}\right)_{t^{k}}+c_{t^{h-k-1}}\left(S_{0}\right)_{t^{k+1}}\right] \\
& =u_{t^{h}}-\sum_{k=2}^{h}\left(\begin{array}{l}
h \\
k
\end{array}\right) c_{t^{h-k}}\left(S_{0}\right)_{t^{k}}-(h-1) c_{t^{h-1}}\left(S_{0}\right)_{t} .
\end{aligned}
$$

Из этого равенства и (4.9) имеем

$$
\left(S_{0,2}\right)_{t}=c_{t^{h-1}}\left(S_{0}\right)_{t}+d S_{0}+u_{2}
$$

Отсюда и из (4.10) следует, что

$$
S_{0}^{-1} S_{0,2}=c_{t^{h-1}}+S_{1},\left(S_{0}^{-1} S_{0,2}\right)_{t}=d+S_{2}
$$

где $S_{1}=S_{0}^{-1}\left(u_{1}\right)_{t^{h-1}}, S_{2}=S_{0}^{-1} u_{2}-S_{0}^{-2}\left(S_{0}\right)_{t}\left(u_{1}\right)_{t^{h-1}}$. Поэтому

$$
I(t)=c_{t^{h-1}}-\int_{0}^{t} d(\tau) d \tau=\int_{0}^{t} S_{2}(x, \tau) d \tau-S_{1}(x, t) .
$$

Так как $u_{t^{h-1}}, u_{2} \in H_{\beta^{\prime}}^{l, 0}\left(K_{T}\right)$, то $S_{1}, S_{2} \in H_{-n / 2}^{0,0}\left(K_{T}\right)$. Следовательно, $I(t) \in$ $H_{-n / 2}^{0}(K)$, т.е. $I(t) \equiv 0$. Отсюда находим, что $c_{t^{h}}=d \in L_{2}(0, T)$ и $\left(u_{1}\right)_{t^{h}}=u_{2} \in$ $H_{m-\beta}^{2 m, 0}\left(K_{T}\right)$. Лемма доказана.

ТеОРема 4.1. Пусть $и$-обобщенное решение задачи (0.2)-(0.4), $|x|>R=$ const, u nycms $f_{t^{k}} \in H_{0}^{l, 0}\left(K_{T}\right)$ npu $k \leqslant 2 l+h+1, f_{t^{k}}(x, 0)=0$ npu $k \leqslant 2 l+h$. Предположим, что прямые

$$
\operatorname{Im} \lambda=m-\frac{n}{2} \quad u \quad \operatorname{Im} \lambda=2 m+l-\frac{n}{2}
$$

не содержат точек спектра задачи (3.6)-(3.7) ни при каких $t \in[0, T]$, а в nолосе

$$
m-\frac{n}{2}<\operatorname{Im} \lambda<2 m+l-\frac{n}{2}
$$

имеется только одно простое собственное значение $\lambda(t)$ задачи (3.6)-(3.7). Тогда справедливо представление

$$
u(x, t)=\sum_{s=0}^{l+m-1} c_{s}(t) r^{-i \lambda(t)+s} P_{3 l+m-1, s}(\ln r)+u_{1}(x, t),
$$

где $P_{3 l+m-1, s}$ - полином степени меньше $3 l+m$, коэффициенты которого есть бесконечно дифференцируемые функиии от $(\omega, t), a\left(c_{s}\right)_{t^{k}} \in L_{2}[0, T] u$ $\left(u_{1}\right)_{t^{k}} \in H_{0}^{2 m+l, 0}\left(K_{T}\right) n p u 0 \leqslant k \leqslant h$.

Далее через $P \ldots$ и $\widetilde{P}_{\ldots}$ б будем обозначать полиномы различных степеней, коэффициенты которых являются бесконечно дифференцируемыми функциями от $(\omega, t)$. 
ДоКАЗАТЕЛЬСТво. Будем использовать метод индукции по $l$. При $l=0$ имеем: $m-n / 2<\operatorname{Im} \lambda(t)<2 m-n / 2$.

Прежде всего докажем, что в случае $m-n / 2<\operatorname{Im} \lambda(t)<m+m_{0}-n / 2$, $1 \leqslant m_{0} \leqslant m$,

$$
u(x, t)=\sum_{s=0}^{m_{0}-1} c_{s}(t) r^{-i \lambda(t)+s} P_{m_{0}-1, s}(\ln r)+u_{1}(x, t),
$$

где $P_{m_{0}-1, s}-$ полином степени меньше $m_{0},\left(c_{s}\right)_{t^{k}} \in L_{2}[0, T],\left(u_{1}\right)_{t^{k}} \in H_{m-m_{0}}^{2 m, 0}\left(K_{T}\right)$ при $k \leqslant h$.

Введем обозначение: $L_{1}=(-1)^{m-1}\left[L_{0}(0, t ; D)-L(x, t ; D)\right]$. Из системы $(0.2)$ имеем

$$
(-1)^{m-1} L_{0}(0, t ; D) u=F,
$$

где $F=u_{t t}+f+L_{1} u$.

Так как $u_{t t}, f \in L_{2}\left(K_{T}\right)$ и $u \in H_{m}^{2 m, 0}\left(K_{T}\right)$, то $F \in H_{m-1}^{0,0}$. Пусть $m-n / 2<$ $\operatorname{Im} \lambda(t)<m+1-n / 2$. Из леммы 4.2 следует, что

$$
u(x, t)=c(t) r^{-i \lambda(t)} \varphi(\omega, t)+u_{1}(x, t),
$$

где $\varphi$ - не зависящая от решения бесконечно дифференцируемая по $\omega, t$ функция, а $c_{t^{k}} \in L_{2}(0, T)$ и $\left(u_{1}\right)_{t^{k}} \in H_{m-1}^{2 m, 0}\left(K_{T}\right)$ при $k \leqslant h$. Итак, $(4.12)$ при $m_{0}=1$ доказано.

Пусть (4.12) верно для $m_{0} \leqslant m-1$. Рассмотрим следующие случаи.

1-й случай: $m-n / 2<\operatorname{Im} \lambda(t)<m+m_{0}-n / 2$. В силу индуктивной гипотезы получим (4.12). Положим

$$
S_{m_{0}}=(-1)^{m} \sum_{s=0}^{m_{0}-1} c_{s}(t) r^{-i \lambda(t)+s} P_{m_{0}-1, s}(\ln r)
$$

Отсюда находим

$$
L S_{m_{0}}=F_{1}(x, t)+\sum_{j+s \leqslant m_{0}} \sum_{s=0}^{m_{0}-1} c_{s}(t) r^{-i \lambda(t)-2 m+s+j} \widetilde{P}_{m_{0}-1, s, j}(\ln r),
$$

где $\left(F_{1}\right)_{t^{k}} \in H_{m-m_{0}-1}^{0,0}\left(K_{T}\right)$ при $0 \leqslant k \leqslant h$, а $\widetilde{P}_{m_{0}-1, s, j}-$ полином степени меньше $m_{0}$.

Из (4.12), (4.13) и (4.16) получаем

$$
\begin{aligned}
& (-1)^{m-1} L_{0}(0, t ; D) u_{1} \\
& \quad=F_{2}(x, t)+\sum_{j+s \leqslant m_{0}} \sum_{s=0}^{m_{0}-1} c_{s}(t) r^{-i \lambda(t)-2 m+s+j} \widetilde{P}_{m_{0}-1, s, j}(\ln r),
\end{aligned}
$$

где $F_{2}=u_{t t}+f+L_{1} u_{1}+F_{1} \in H_{m-m_{0}-1}^{0,0}\left(K_{T}\right)$. 
В силу леммы 4.1 функция

$$
w_{1}=\sum_{j+s \leqslant m_{0}} \sum_{s=0}^{m_{0}-1} c_{s}(t) r^{-i \lambda(t)+s+j} P_{m_{0}, s, j}(\ln r)
$$

такова, что

$$
\begin{aligned}
& (-1)^{m-1} L_{0}(0, t ; D) w_{1} \\
& \quad=\sum_{j+s \leqslant m_{0}} \sum_{s=0}^{m_{0}-1} c_{s}(t) r^{-i \lambda(t)-2 m+s+j} \widetilde{P}_{m_{0}-1, s, j}(\ln r),
\end{aligned}
$$

где $P_{m_{0}, s, j}$ - полином степени менњше $m_{0}$.

Положим $v_{1}=u_{1}-w_{1}$. Из (4.17) и (4.19) следует, что

$$
(-1)^{m-1} L_{0}(0, t ; D) v_{1}=F_{2}(x, t)
$$

С помощью леммы 4.2 получим

$$
v_{1}(x, t)=c_{0}(t) r^{-i \lambda(t)} \varphi(\omega, t)+u_{2}(x, t),
$$

где $\varphi$ - не зависяшая от решения бесконечно дифференцируемая функция своих аргументов, а $\left(u_{2}\right)_{t^{k}} \in H_{m-m_{0}-1}^{2 m, 0}\left(K_{T}\right)$ при $0 \leqslant k \leqslant h$.

Из (4.18) и (4.20) имеем

$u_{1}(x, t)=c_{0}(t) r^{-i \lambda(t)} \varphi(\omega, t) \sum_{j+s \leqslant m_{0}} \sum_{s=0}^{m_{0}-1} c_{s}(t) r^{-i \lambda(t)+s+j} P_{m_{0}, s, j}(\ln r)+u_{2}(x, t)$.

Отсюда и из (4.12) находим

$$
u(x, t)=\sum_{s=0}^{m_{0}} \tilde{c}_{s}(t) r^{-i \lambda(t)+s} \widetilde{P}_{m_{0}, s}(\ln r)+u_{2}(x, t),
$$

где $\widetilde{P}_{m_{0}, s}-$ полином степени менњше $m_{0}+1$, а $\left(\tilde{c}_{s}\right)_{t^{k}} \in L_{2}[0, T]$ и $\left(u_{2}\right)_{t^{k}} \in$ $H_{m-m_{0}-1}^{2 m, 0}\left(K_{T}\right)$ при $0 \leqslant k \leqslant h$.

2-й случай: $m+m_{0}-n / 2<\operatorname{Im} \lambda(t)<m+m_{0}+1-n / 2$. Так как в полосе $m-n / 2 \leqslant \operatorname{Im} \lambda(t) \leqslant m+m_{0}-n / 2$ нет собственных значений задачи (3.6)-(3.7), то $u \in H_{m-m_{0}}^{2 m, 0}\left(K_{T}\right)$. С другой стороны, $m+m_{0}-n / 2<\operatorname{Im} \lambda(t)<m+m_{0}+1-n / 2$. Следовательно, из леммы 4.2 следует, что

$$
u(x, t)=c(t) r^{-i \lambda(t)} \varphi(\omega, t)+u_{1}(x, t),
$$

где $\varphi$ - не зависяшая от решения бесконечно дифференцируемая функция, $c_{t} \epsilon \in$ $L_{2}(0, T)$ и $\left(u_{1}\right)_{t^{k}} \in H_{m-m_{0}-1}^{2 m, 0}\left(K_{T}\right)$ при $k \leqslant h$.

3 -й случай: существует $t_{0}$ такое, что $\operatorname{Im} \lambda\left(t_{0}\right)=m+m_{0}-n / 2$. Можно считать, что $m+m_{0}-\mu-n / 2<\operatorname{Im} \lambda(t)<m+m_{0}-\mu+1-n / 2,0<\mu<1$. Повторяя рассуждения доказательства 2-го случая, получим (4.22), где $\left(u_{1}\right)_{t^{k}} \in H_{m-m_{0}-1+\mu}^{2 m, 0}\left(K_{T}\right)$ при $k \leqslant h$. Отсюда аналогично доказательству 1 -го случая получим (4.21). 
Итак, (4.12) доказано, т.е. утверждение теоремы верно для $l=0$.

Допустим, что утверждение теоремы верно при $l$. Пусть $m-n / 2<\operatorname{Im} \lambda(t)<$ $2 m+l-n / 2$. Из индуктивной гипотезы имеем (4.11). Следовательно,

$$
(-1)^{m-1} L_{0}(0, t ; D) u_{1}=F_{3}+(-1)^{m} L S-S_{t t},
$$

где $F_{3}=\left(u_{1}\right)_{t t}+f+L_{1} u_{1}, \mathrm{a}$

$$
S=\sum_{s=0}^{l+m-1} c_{s}(t) r^{-i \lambda(t)+s} P_{3 l+m-1, s}(\ln r)
$$

Так как $f_{t^{k}} \in H_{0}^{l+1,0}\left(K_{T}\right), k \leqslant 2(l+1)+h+1$, то $\left(c_{s}\right)_{t^{k}} \in L_{2}(0, T)$ и $\left(u_{1}\right)_{t^{k}} \in$ $H_{0}^{2 m+l, 0}\left(K_{T}\right)$ при $k \leqslant h+2$. Следовательно, $\left(F_{3}\right)_{t^{k}} \in H_{0}^{l+1,0}\left(K_{T}\right)$. С другой стороны, имеем

$$
(-1)^{m} L S-S_{t t}=F_{4}+\sum_{s=0}^{l+m} \tilde{c}_{s}(t) r^{-i \lambda(t)-2 m+s} \widetilde{P}_{3 l+m+1, s}(\ln r)
$$

где $\widetilde{P}_{3 l+m+1, s}-$ полином степени меньше $3 l+m+2$, a $\left(F_{4}\right)_{t^{k}} \in H_{0}^{l+1,0}\left(K_{T}\right)$ и $\left(\tilde{c}_{s}\right)_{t^{k}} \in L_{2}(0, T)$. Поэтому из $(4.23)$ получим

$$
(-1)^{m-1} L_{0}(0, t ; D) u_{1}=F_{5}+\sum_{s=0}^{l+m} \tilde{c}_{s}(t) r^{-i \lambda(t)-2 m+s} \widetilde{P}_{3 l+m+1, s}(\ln r)
$$

где $F_{5}=F_{3}+F_{4} \in H_{0}^{l+1,0}\left(K_{T}\right) \subseteq H_{-1}^{l, 0}\left(K_{T}\right)$. В силу леммы 4.2 , рассуждая, как при доказательстве 1-го случая, из (4.24) находим

$$
u_{1}(x, t)=\sum_{s=0}^{l+m} \tilde{c}_{s}(t) r^{-i \lambda(t)+s} \widetilde{P}_{3 l+m+2, s}(\ln r)+u_{2}(x, t)
$$

где $\widetilde{P}_{3 l+m+2, s}$ - полином степени меньше $3 l+m+3$, a $\left(u_{2}\right)_{t^{k}} \in H_{-1}^{2 m+l, 0}\left(K_{T}\right)$ при $k \leqslant h$. С помошью леммы 3.2 имеем: $\left(u_{2}\right)_{t^{k}} \in H_{0}^{2 m+l+1,0}\left(K_{T}\right), k \leqslant h$. Отсюда и из (4.11) следует, что

$$
u(x, t)=\sum_{s=0}^{l+m} c_{s}(t) r^{-i \lambda(t)+s} P_{3 l+m+2, s}(\ln r)+u_{2}(x, t)
$$

где $P_{3 l+m+2, s}-$ полином степени меньше $3 l+m+3$, а $\left(c_{s}\right)_{t^{k}} \in L_{2}[0, T]$ и $\left(u_{2}\right)_{t^{k}} \in$ $H_{0}^{2 m+l+1,0}\left(K_{T}\right)$ при $0 \leqslant k \leqslant h$.

Рассуждая так же, как и при доказательстве 2-го и 3-го случаев, можно доказать, что если $2 m+l-n / 2<\operatorname{Im} \lambda(t)<2 m+l+1-n / 2$ или существует $t_{0}$ такое, что $\operatorname{Im} \lambda\left(t_{0}\right)=2 m+l-n / 2$, то справедливо представление (4.26). Отсюда получим утверждение теоремы для $l+1$. Теорема доказана. 
TЕОРема 4.2. Пусть $и$ - обобщенное решение задачи (0.2)-(0.4), $|x|>R=$ const, $u$ nycms $f_{t^{k}} \in H_{0}^{l, 0}\left(K_{T}\right)$ npu $k \leqslant 2 l+h+1, f_{t^{k}}(x, 0)=0$ npu $k \leqslant 2 l+h$. Предполохсим, что прямые

$$
\operatorname{Im} \lambda=m-\frac{n}{2} \quad u \quad \operatorname{Im} \lambda=2 m+l-\frac{n}{2}
$$

не содержат точек спектра задачи (3.6)-(3.7) ни при каких $t \in[0, T]$, а в noлосе

$$
m-\frac{n}{2}<\operatorname{Im} \lambda<2 m+l-\frac{n}{2}
$$

лежсат только простые собственные значения $\lambda_{1}(t), \lambda_{2}(t), \ldots, \lambda_{N_{0}}(t)$ задачи (3.6)-(3.7) такие, что $\operatorname{Im} \lambda_{1}(t)<\operatorname{Im} \lambda_{2}(t)<\cdots<\operatorname{Im} \lambda_{N_{0}}(t), \operatorname{Im} \lambda_{j}(t) \neq$ $\operatorname{Im} \lambda_{k}(t)+N, j \neq k, N-$ uелое, $j, k=1, \ldots, N_{0}, t \in[0, T]$. Тогда справедливо представление

$$
u(x, t)=\sum_{j=1}^{N_{0}} \sum_{s=0}^{l+m-1} c_{s, j}(t) r^{-i \lambda_{j}(t)+s} P_{3 l+m-1, s, j}(\ln r)+u_{1}(x, t),
$$

где $P_{3 l+m-1, s, j}$ - полином степени меньие $3 l+m$, коэффициенты которого являются бесконечно дифференцируемыми по $\omega, t$ функииями, $a\left(c_{s, j}\right)_{t^{k}} \in$ $L_{2}[0, T] u\left(u_{1}\right)_{t^{k}} \in H_{0}^{2 m+l, 0}\left(K_{T}\right)$ npu $0 \leqslant k \leqslant h$.

ДокАЗАТЕЛЬСтво. При любом $t_{0} \in(0, T)$ существует $\varepsilon>0$ такое, что $m+$ $\mu_{j-1}-n / 2<\operatorname{Im} \lambda_{j}(t)<m+\mu_{j}-n / 2, t \in\left[t_{0}-\varepsilon, t_{0}+\varepsilon\right], \mu_{j}=$ const $\geqslant 0$, $j=0,1, \ldots, N_{0}$. Так как отрезок $[0, T]$ является компактным множеством, то существуют числа $T_{0}=0, T_{1}, \ldots, T_{M-1}, T_{M}=T$ такие, что $m+\mu_{j-1, s}-n / 2<$ $\operatorname{Im} \lambda_{j}(t)<m+\mu_{j, s}-n / 2, t \in\left[T_{j-1}, T_{j}\right], \mu_{j, s}=$ const $\geqslant 0, j=1, \ldots, N_{0}, s=$ $0,1, \ldots, M$. Без ограничения общности можно считать, что $m-n / 2<\operatorname{Im} \lambda_{1}(t)<$ $m+\mu_{1}-n / 2<\operatorname{Im} \lambda_{2}(t)<\cdots<m+\mu_{N_{0}-1}-n / 2<\operatorname{Im} \lambda_{N_{0}}(t)<2 m+l-n / 2$, $t \in[0, T]$.

Для доказательства теоремы воспользуемся методом индукции по $N_{0}$. При $N_{0}=1$ утверждение теоремы следует из теоремы 4.1. Пусть утверждение теоремы верно для $N_{0}-1$. Для простоты считаем, что $\mu_{N_{0}-1}=l_{0}<l$. Из индуктивной гипотезы получим

$$
u(x, t)=\sum_{j=1}^{N_{0}-1} \sum_{s=0}^{l_{0}+m-1} c_{s, j}(t) r^{-i \lambda_{j}(t)+s} P_{3 l_{0}+m-1, s, j}(\ln r)+u_{1}(x, t),
$$

где $P_{3 l_{0}+m-1, s, j}-$ полином степени меньше $3 l_{0}+m, \mathrm{a}\left(c_{s, j}\right)_{t^{k}} \in L_{2}[0, T]$ и $\left(u_{1}\right)_{t^{k}} \in$ $H_{0}^{2 m+l_{0}, 0}\left(K_{T}\right)$ при $0 \leqslant k \leqslant h$. Рассуждая, как при доказательстве $(4.24)$, получим

$$
\begin{aligned}
& (-1)^{m-1} L_{0}(0, t ; D) u_{1} \\
& =\widetilde{F}+\sum_{j=1}^{N_{0}-1} \sum_{s=0}^{l_{0}+m} \tilde{c}_{s, j}(t) r^{-i \lambda_{j}(t)-2 m+s} \widetilde{P}_{3 l_{0}+m+1, s, j}(\ln r),
\end{aligned}
$$


где $\widetilde{F} \in H_{0}^{l_{0}+1,0}\left(K_{T}\right), \widetilde{P}_{3 l_{0}+m-1, s, j}$ - полином степени меньше $3 l_{0}+m, \mathrm{a}\left(\tilde{c}_{s, j}\right)_{t^{k}} \in$ $L_{2}[0, T], 0 \leqslant k \leqslant h$. Отсюда следует, что если $2 m+l_{1}-n / 2<\operatorname{Im} \lambda_{N_{0}}(t)<2 m+$ $l_{1}+1-n / 2, l_{1} \geqslant l_{0}$, то

$$
u_{1}(x, t)=\sum_{j=1}^{N_{0}} \sum_{s=0}^{l_{1}+m} \tilde{c}_{s, j}(t) r^{-i \lambda_{j}(t)+s} \widetilde{P}_{3 l_{1}+m+2, s}(\ln r)+u_{2}(x, t)
$$

где $\widetilde{P}_{3 l_{1}+m+2, s}$ - полином степени меньше $3 l_{1}+m+3$, a $\left(u_{2}\right)_{t^{k}} \in H_{0}^{2 m+l_{1}+1,0}\left(K_{T}\right)$, $k \leqslant h$.

Так как в полосе $2 m+l_{1}+1-n / 2 \leqslant \operatorname{Im} \lambda \leqslant 2 m+l-n / 2$ нет собственных значений задачи (3.6)-(3.7), то из (4.28)-(4.30) получаем (4.27).

Если существует $t_{0}$ такое, что $\operatorname{Im} \lambda\left(t_{0}\right)=2 m+l_{1}-n / 2$, то в силу леммы 3.2 аналогично доказательству 3 -го случая теоремы 4.1 получим (4.27). Теорема доказана.

Переходим к асимптотике обобшенного решения первой краевой задачи для сильно гиперболических систем в ограниченных областях с конической точкой на границе. Применяя такие же рассуждения, как и при доказательстве теоремы 3.3, из теоремы 4.2 получим следующую теорему.

ТЕОРема 4.3. Пусть $и$ - обобщенное решение задачи (0.2)-(0.4), и пусть $f_{t^{k}} \in H_{0}^{l, 0}\left(\Omega_{T}\right) n p u k \leqslant 2 l+h+1, f_{t^{k}}(x, 0)=0$ nрu $k \leqslant 2 l+h$. Пусть, кроме того, прямые $\operatorname{Im} \lambda=m-n / 2 u \operatorname{Im} \lambda=2 m+l-n / 2$ не содерхат точек спектра задачи (3.6)-(3.7) ни при каких $t \in[0, T]$, а в полосе

$$
m-\frac{n}{2}<\operatorname{Im} \lambda<2 m+l-\frac{n}{2}
$$

лехат простые собственнье значения $\lambda_{1}(t), \ldots, \lambda_{N_{0}}(t)$ задачи (3.6)-(3.7) такие, что $\operatorname{Im} \lambda_{1}(t)<\operatorname{Im} \lambda_{2}(t)<\cdots<\operatorname{Im} \lambda_{N_{0}}(t), \operatorname{Im} \lambda_{j}(t) \neq \operatorname{Im} \lambda_{k}(t)+N, j \neq k$, $N$ - челое, $j, k=1, \ldots, N_{0}, t \in[0, T]$. Тогда в окрестности конической точки справедливо представление

$$
u(x, t)=\sum_{j=1}^{N_{0}} \sum_{s=0}^{l+m-1} c_{s, j}(t) r^{-i \lambda_{j}(t)+s} P_{3 l+m-1, s, j}(\ln r)+u_{1}(x, t),
$$

где $P_{3 l+m-1, s, j}$ - полином степени меньше $3 l+m$, коэффициенть которого являются бесконечно дифференцируемыми по $\omega, t$ функциями, $a\left(c_{s, j}\right)_{t^{k}} \in$ $L_{2}[0, T] u\left(u_{1}\right)_{t^{k}} \in H_{0}^{2 m+l, 0}\left(\Omega_{T}\right)$ npu $0 \leqslant k \leqslant h$.

Автор выражает искреннюю благодарность В.А. Кондратьеву за постановку задачи и внимание к работе. 


\section{Список литературы}

1. Kreiss H. O. Initial boundary value problem for hyperbolic systems // Comm. Pure Appl. Math. 1970. V. 3. P. 277-298.

2. Сакамото Р. Смешанные задачи для гиперболических уравнений // Математика. 1972. T. 16. № 1. C. $62-80$.

3. Блохин А. М., Ткачев Д. Л. Смешанная задача для волнового уравнения в области с углом (скалярный случай) // Сиб. матем. журн. 1989. Т. 15. № 3. С. 16-23.

4. Пламеневский Б. А. О волновом уравнении в цилиндре с ребрами // Функц. анализ и его прилож. 1998. Т. 32. №1. С. 81-84.

5. Eskin G. The wave equation in a wedge with general boundary conditions // Comm. Partial Differential Equations. 1992. V. 17. № 1, 2. P. 99-160.

6. Ладызсенская О.А. О нестационарных операторных уравнениях и их приложениях к линейньм задачам математической физики // Матем. сб. 1958. Т. 45 (87). № 2. C. $123-158$.

7. Лады жсенская О. А. Краевые задачи математической физики. М.: Наука, 1973.

8. Мельников И. И. Особенности решения смешанной задачи для гиперболических уравнений второго порядка в областях с кусочно гладкой границей // УМН. 1982. Т. 37. № 1. C. $149-150$.

9. Наваль Сайед Ахмед Шериф. Смешанная задача для гиперболических уравнений в областях с негладкой границей // Вестн. МГУ. Сер. 1. Матем., мех. 1980. № 3. С. 7-10.

10. Фикера Г. Теоремы существования в теории упругости. М.: Мир, 1974.

11. Агмон C., Дуглис А., Ниренберг Л. Оценки вблизи границы решений эллиптических уравнений в частных производных при общих граничных условиях. І. М.: ИЛ, 1962.

12. Мазья В.Г., Пламеневский Б. А. О коэффициентах в асимптотике решений эллиптических краевых задач в областях с коническими точками // Math. Nachr. 1977. V. 76. P. 29-60.

13. Назаров С.А., Пламеневский Б. А. Эллиптические задачи в областях с кусочно гладкой границей. М.: Наука, 1991.

14. Ени B. М. Об устойчивости корневого числа аналитической оператор-функции и о возмущениях ее характеристических чисел и собственных векторов // Докл. АН СССР. 1967. T. 173. №6. C. 1251-1254.

Московский государственньй

Поступила в редакцию университет им. М. В. Ломоносова

18.08.1997 и 20.01.1999 